\title{
Validation of low-cost wireless EEG system for measuring event-related potentials
}

\author{
Yongxiang Wang \\ Department of Engineering \\ Institute of Technology Blanchardstown \\ Dublin 15, Ireland \\ yongxiang.wang@mydit.ie \\ Charles Markham \\ Department of Computer Science \\ National University of Ireland \\ Maynooth, Ireland \\ charles.markham@mu.ie
}

\author{
Zhengwei Wang \\ Insight Centre for Data Analytics \\ Dublin City University \\ Dublin 9, Ireland \\ zhengwei.wang22@mail.dcu.ie \\ Tomas E. Ward \\ Insight Centre for Data Analytics \\ Dublin City University \\ Dublin 9, Ireland \\ tomas.ward@dcu.ie
}

\author{
William Clifford \\ Department of Computer Science \\ National University of Ireland \\ Maynooth, Ireland \\ willam.clifford.2012@mumail.ie
}

\author{
Catherine Deegan \\ Department of Engineering \\ Institute of Technology Blanchardstown \\ Dublin 15, Ireland \\ catherine.deegan@itb.ie
}

\begin{abstract}
This study used the traditional P300 speller paradigm to compare a medical grade Electroencephalography (EEG) system, the G.Tec, with a consumer grade EEG system, the Emotiv, in the detection of P300 components within Event Related Potential (ERP) signals. The experiment focused on four electrodes known to produce optically induced visual evoked potential. A successful comparison of the two approaches was made. It was shown that both systems could measure an ERP. The paper concludes with discussion comparing the low-cost wireless EEG system with the medical grade EEG system.
\end{abstract} G.Tec

Keywords-EEG, ERP, Evoked potential, P300, N200, Emotiv,

\section{INTRODUCTION}

Probably the most common neuroelectric technique to measure human brain activities is Electroencephalography (EEG), a noninvasive measurement, made with electrodes placed on the scalp. EEG is most often used to diagnose diseases of the brain such as seizure disorders, memory problems and sleep disorders $[1,2]$. The electrical activity on the surface of the head measured by EEG can be analysed in terms of frequency (e.g. Alpha, Beta brainwaves) and Event Related Potential (ERP). ERP is used to investigate brain activity synchronised to a time-locked stimulus or physical activity in the human body [3-5]. This paper focuses on the potential of making measurements of ERPs using a consumer grade EEG. Current EEG research is focused on developing brain computer interfaces (BCI) for both gaming and assisting people with disability.The protocols used for this experiment make use of the technique developed for EEG spellers.

In the past decade, EEG-based BCI have been the focus of research in application areas used to spell words, control an electronic device, or complete a task in a gaming application [6].

Until recently, the majority of equipment used to measure EEG, have been traditional medical grade devices $[1,2,4]$. These devices have their strengths and weaknesses. The most significant advantage of this approach is the availability of high precision time and potential measurements. Whether the participant is awake or asleep, the brain is constantly generating a variety of electrical signals to control the

This work is funded by Dublin Institute of Technology Fiosraigh Scholarship Program. functioning of the body. The changes in these electrical activities are typically measured in millisecond timescales. Therefore, high time resolution is necessary to determine the precise moments at which these electrical events take place. EEG has been shown to provide an effective way of measuring visual ERP's in experiments. In a working memory (WM) and psychomotor vigilance task (PVT) reported in the literature, the authors show that an effective reliability measure can be achieved in both cases sessions ( $r>0.9$ in WM tasks and $r>$ 0,8 in PVT task) [6]. In another study, the authors demonstrate that similar reliability can be achieved in response to ERPS generated by face and noise textures $(\mathrm{r} \sim 0.9)[7]$.

The disadvantages of traditional EEG devices are limited to wired connections to each individual scalp electrodes attached to the amplifier [1]; the devices can take a long time to adjust the electrodes to get a good signal; It is also required to use conductive gel on individual electrodes to ensure good conduction to scalp. The wired connection and gel electrodes create a restriction to movement, in order to achieve a clean EEG signal. In addition, a complete EEG system can also be quite costly. In recent years, a number of consumer grade EEG devices have been developed and commercialised. These devices specify themselves as BCI devices and wearable meditation headsets [7-9]. The advantageous features of these devices are low-cost, wireless and portable. Researchers were gained interests in such devices as their potential to perform comparable EEG measurement compared with the medical grade EEG systems inside and outside the laboratory [10-12].

The most popular consumer grade EEG devices are the NeuroSky MindWave ${ }^{\mathrm{TM}}$ [9], the NeuroFocus Mynd ${ }^{\mathrm{TM}}$, the Muse $^{\mathrm{TM}}$ [7] and the Emotiv EPOC ${ }^{\mathrm{TM}}$ [8]. These devices only need minor adjustment for setup and typically use dry electrodes or saline soaked cotton pads in direct contact with the scalp. This quick setup coupled with wireless communication makes them ideal for studies using a larger number of participants as it reduces disruption to the wearer.

Although there has been an increase in the use of low-cost EEG devices in research applications, the question of measured EEG quality still remains. The Emotiv EPOC has been given the most attention in the literature compared to other wireless EEG systems [11, 15-20]. 
Campbell et al. [10] developed a NeuroPhone system on a smartphone similar to a P300 speller experiment. The P300 wave is a positive deflection in ERP and it is most likely elicited from oddball paradigm when subject detects an occasional 'Target' stimulus [4, 13]. The NeuroPhone system has a specially designed phone book that has six photos flashed in sequence, the user looked at one photo and used an Emotiv EPOC headset to record user's brain activity, then analysed the EEG data in real time to detect which photo the user wished to dial. The paper reported a good accuracy of P300 signal detection under sitting conditions (percentage of all events that are correctly classified as P300 component, accuracy is $88.89 \%$ ). The paper also suggests that P300 signals are susceptible to external noise. It was necessary to average a large number of trials to obtain a reliable P300 signal. Tong et al. [11] came up with a similar P300 based dialing BCI system on a tablet and used the Emotiv EPOC to detect P300 signals. The system used $4 \times 3$ matrices of alphanumeric characters that enabled users to spell a phone number. The system has been tested in a relatively quiet lab/office environment and noisy subway environment. The results showed similar findings that using the system in a noisy environment produced a low performance (accuracy in a laboratory is $88.75 \pm 10.57$ and in a subway environment 73.75 \pm 16.94$)$. Debener et al. [12] modified the Emotiv EPOC to reconnect all electrodes onto a EEG cap with $\mathrm{Ag} / \mathrm{AgCl}$ electrodes instead of saline soaked cotton pad at location FPz, F3, Fz, F4, C3, Cz, C4, TP9, Tp10, $\mathrm{P} 3, \mathrm{Pz}, \mathrm{P} 4, \mathrm{O} 1$, and $\mathrm{O} 2$ according to $10-20$ system. Their study used an auditory oddball paradigm presented to participants with standard $(n=660)$ and deviant $(n=84)$ stimulus in indoor and outdoor conditions. Debener et al. found that the deviant stimulus produced a higher amplitude P300 signal in both indoor and outdoor conditions compared to standard stimulus. In addition, indoor conditions produced significantly larger P300 signals.

The research papers reviewed above demonstrated that the Emotiv EPOC can detect P300 signals. However, comparison of the performance of the Emotiv with other, research grade systems has not been widely available. Duvinage et al. compared the Emotiv EPOC and Advanced Neuro Technology (ANT) acquisition system by using a modified P300 speller task, which has only four alphabet letters to detect visual evoked potentials [17]. Participants wore two devices separately in different sessions. Both the Emotiv EPOC and ANT acquisition system obtained reliable P300 signals. However, the Emotiv system has weaker P300 signal detection due to a poorer intrinsic signal to noise ratio (SNR). Badcock et al. $[14,15]$ and Lissa et al. [16] conducted three studies that compared the Emotiv EPOC and Neuroscan system. The authors proposed a novel approach, which placed two devices side by side according to the classic 10-20 system location in order to let both systems record EEGs simultaneously. The early study from Badcock et al. was presented with an auditory oddball stimulus to adults with deviant tones $(n=100)$ and standard tones $(n=566)$ played randomly [16]. The authors carried out a list of comparison results with the electrodes in the frontal lobe (A3, A4, AF3, AF4). In this work, they showed that the Emotiv produced a slightly less reliable signal than the Neuroscan system. Both systems detected an ERP. The later study of Badcock et al. [14] replicated the same experiment with 18 children. The results produced were remained very similar, both the Neuroscan system and the Emotiv system detected standard and deviant waves with high reliability. Stytsenko et al. provided a comparison between Emotiv EPOC and G.Tec system also confirmed that both systems are alike in general, but the signal is cleaner and stronger in the G.Tec system [17].

The aim of this paper is to use the traditional P300 speller experiment to evaluate the performance of Emotiv EPOC+ device compared to medical grade G.Tec g.USBamp system [18]. Demonstration of the effectiveness of the wireless system will allow its incorporation into a driving simulator to assess the driver's cognitive response to distraction events synchronised with an eye-tracking system.

\section{Methodology}

\section{A. Participants}

All measurements were taken from three healthy individuals (age range: 23 to 53 years) free of past or present neurological or psychiatric conditions with normal or corrected to normal vision. Small participant numbers in these types of studies is not unusual $[10,19,20]$. As this is an initial commissioning experiment to validate a wireless EEG device, a small sample size is deemed sufficient to characterise the signal response of the device itself.

\section{B. Visual oddball task}

The participants were required to attend the most widely used visual oddball task, the "P300 speller", designed by Farwell and Donchin [21]. The speller is a 6x6 matrix, consisting of 26 alphabet letters and 10 digital numbers (0-9) as shown in Fig. 1. The participants' task was to focus on ten randomly selected symbols in the matrix one at a time. The row and column of the matrix were randomly intensified for 200 milliseconds (ms), followed by $100 \mathrm{~ms}$ of nonintensification. Two out of twelve intensifications contained the target symbol $(16.7 \%$ target stimuli and $83.3 \%$ non-target stimuli). Twelve repetitions for each symbol to increase the low SNR due to other un-related brain signals, muscular and ocular artefacts. The inter-repetition delay was set at 1 second. The inter-trial delay was set at 3 seconds. During the inter-trial delay, the task indicated the next highlighted symbol to be focused by the participants.

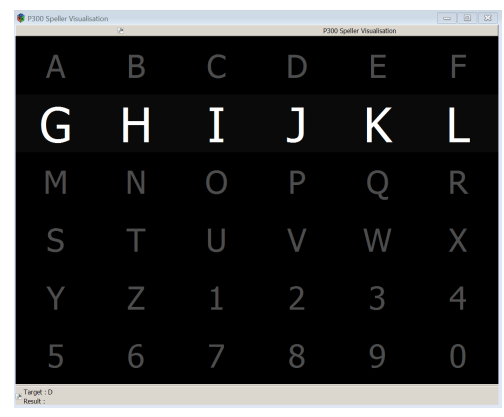

Fig. 1 P300 Interface in OpenViBE [22]. 6x6 matrix used in the experiment. A row or colume intensifies by a given rate. The target letter displays at the bottom of the window. A P300 signal should be elicited when participant see the desired letter is intetensified. 


\section{Equipment}

As participants looked at the desired target symbol, EEG was recorded from a medical grade G.Tec g.USBamp system and a wireless Emotiv system separately. The g.USBamp system uses a standard USB 2.0 interface, which makes the amplifier easily connect to the USB socket on PC/notebook and can immediately be used for data recording. The system has 16 simultaneously sampled biosignal channels with 24 bits resolution. Regarding the wide-range DC-coupled amplifier, the sampling rate can adjust from $64 \mathrm{~Hz}$ to $38.400 \mathrm{~Hz}$. In this experiment, the electrode impedance was measured and maintained under 20 kilohms for each channel using conductive gel. Signals were checked using the G.Tec developed Matlab Simulink impedance checker. Electrode locations were chosen to match the same locations provided by the Emotiv EPOC. In addition, the extra two electrodes were situated at $\mathrm{Pz}$ and $\mathrm{POz}$ locations. The g.USBamp system amplifier has an input range of $\pm 250 \mathrm{mV}$, which allows recording of DC signal without saturation, and has four independent grounds to guarantee no interference between the recorded signals.

The Emotiv EPOC+ headset (2016 later edition), which was used in this experiment, comes with 14 channels and uses CMS/DRL (common mode sense/driven right leg) references at P3/P4 locations. The electrodes are held with a plastic arm that holds a small cap with saline soaked felt pad inside. The electrode locations are based on the international 10-20 system as shown in Fig. 2. The device uses a Bluetooth transmitter and has an inbuilt battery with a life of about 12 hours. The signal is internally digitized at $2048 \mathrm{~Hz}$ (16-bit) and subsequently low pass filtered $(43 \mathrm{~Hz})$ and downsampled to either $128 \mathrm{~Hz}$ or 256 $\mathrm{Hz}$.
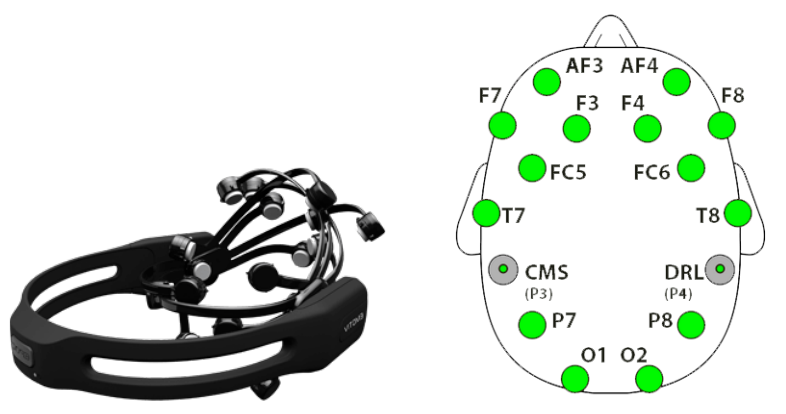

Fig. 2 Emotiv EPOC+ wireless headset (left) [5] and its correspondent electrodes locations in international 10-20 system (right).

\section{EEG recoding}

The experiment took place in a RF shielded room $(>100 \mathrm{~dB}$ $1 \mathrm{GHz}$ and $100 \mathrm{GHz}$ ). Participants sat on a comfortable chair with a 19-inch monitor placed at about one meter in front, as shown in Fig. 3. When recording the EEG, the G.Tec system amplifier and the Emotiv bluetooth dongle were connected to a desktop computer running the OpenViBE open-source software (version 2.0) the experimental runs for each device implemented one after the other [25]. For the G.Tec system we recorded the EEG at $512 \mathrm{~Hz}$ sampling rate and used the left ear lobe as reference, ground electrode was positioned in the forehead at AFz. For Emotiv EPOC+, the reference sensor was at its default location as shown in Fig. 2 and recorded EEG at $128 \mathrm{~Hz}$ sampling rate.

\section{E. Offline EEG processing}

All EEG data recorded from G.Tec g.USBamp and Emotiv systems were processed offline using EEGLAB (v 14.1.1) [23] and ERPLAB (v 7.0) [24]. The first step was downsampling the continuous G.Tec data from 512 to $128 \mathrm{~Hz}$, in order to match the sampling of Emotiv system. The data from the G.Tec and Emotiv were bandpass filtered at $0.16-30 \mathrm{~Hz}$ to remove DC and other high frequency noise. Data from both systems then extracted epochs from $-200 \mathrm{~ms}$ to $800 \mathrm{~ms}$ regards to stimulus onset. Each epoch then was baseline corrected from $-200 \mathrm{~ms}$ to $0 \mathrm{~ms}$ preceding stimulus onset. An automatic epoch rejection method was used to remove extreme value epochs. Independent component analysis (ICA) was used on the epoched data to remove eye blink artefact and facial muscle movement artefacts. For each participant, epoched data comprising target and non-target trials were averaged separately to create ERP waveforms. Electrodes were chosen at channel P7 P8 O1 and $\mathrm{O} 2$ to observe in the experiment, due to the visual cortex is located in the occipital lobe of the brain $[11,25]$. P300 peak latency was assessed between $250 \mathrm{~ms}$ to $550 \mathrm{~ms}$, N200 peak latency was assessed within $160 \mathrm{~ms}$ to 300 $\mathrm{ms}$, using the approach described in the literature [29]. The amplitudes of two components were measured.

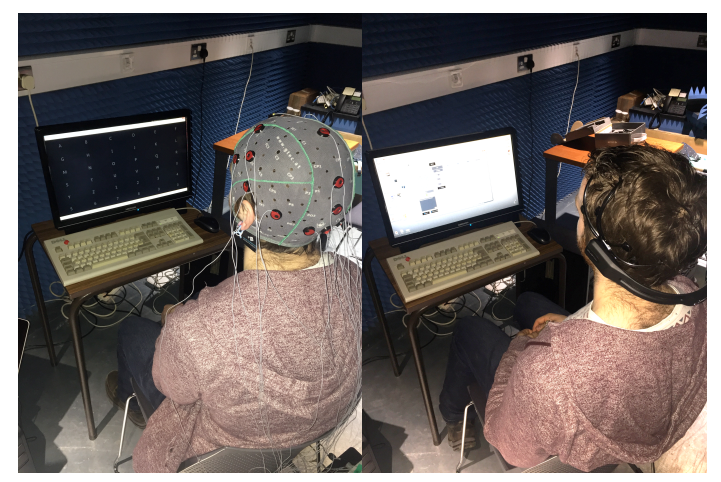

Fig. 3 Experiment setup. The traditional EEG system G.Tec g.USBamp (left) uses cables to connect the cap to the recording machine. The EMOTIV EPOC+ (right) uses Bluetooth wirelessly transmitted signal to the recording machine. The setup of electrode locations, participant sit position, P300 Speller presentation computer monitor position and all parameters potentially influencing the experiment were kept same for two conditions.

\section{RESUlTS}

\section{A. Temporal patterns of ERP components in P300 Speller}

All data epochs were averaged from all trials of each individual electrode of each participant. The correspondent target stimulus epochs and non-target stimulus epochs were averaged respectively.

Fig. 4 presents the single trials ERP image of target stimuli elicited by the P300 speller paradigm from participant $2 \& 3$, electrode location $\mathrm{O} 2$ with the G.Tec system. Fig. 4a shows that the P300 ERP signal is presented in most of the events that include a target stimulus, as seen by the red band around 300 ms. This P300 component shows a constant visual stimulus onset related activity throughout the duration of the experiment. However, over the duration of the experiment, the 
amplitude of the P300 ERP signal was reducing, as well as the latency was varying. The variability of amplitudes and latencies might be affected by the decline in concentration level and the reduced attentional focus. On the left side of the P300 component, a less visible N200 component was also noted, as seen by the blue band at $200 \mathrm{~ms}$. The N200 typically evoked $180 \mathrm{~ms}$ to $325 \mathrm{~ms}$ following the presenting of a specific visual or auditory stimulus [26]. A number of studies have validated that the N200 component can be elicited from an oddball detection task $[14,15,27,28]$. Analysis of evoked potentials in response to visual stimuli reveals a stronger negative component around $220 \mathrm{~ms}$ post-stimulus onset in Fig. $4 \mathrm{~b}$. This N200 component was attributed to more than twothirds of the events that include a target stimulus. In addition, the P300 ERP signal in Fig. 4b is significantly weaker compare to Fig. 4a. A visual N200 experiment demonstrated in [29], discovered that the P300 elicitation can be inhibited by the appearance of N200 in tasks of motor activity suppression. Therefore, we discovered low visibility of P300 components in Fig. 4b may heavily impact by the robust N200 components. Apart from this, subject to subject variability could also directly influence the results.
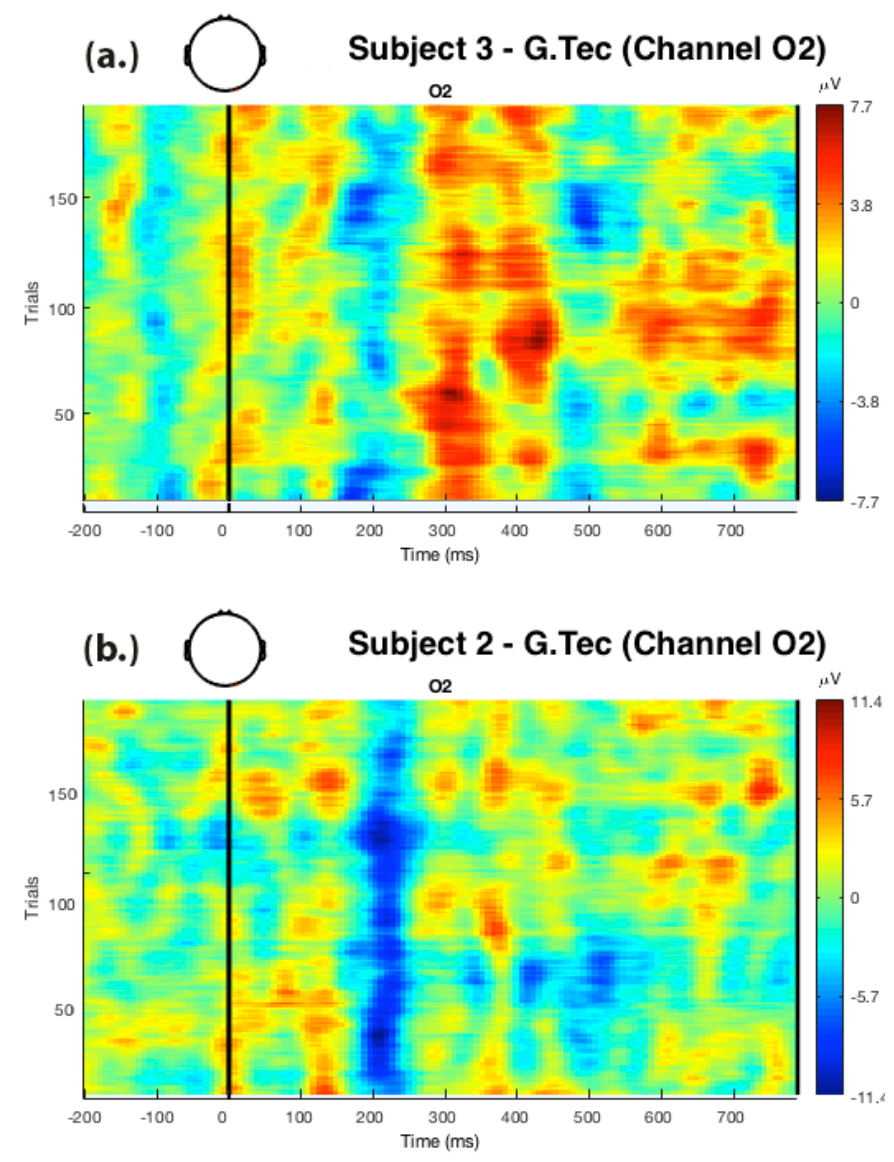

Fig. 4 Single trials ERP images of target stimuli. (a.) This data was recorded with G.Tec system, channel O2 from participant 3. The P300 component can be observed. (b.) This data was recorded with G.Tec system, channel O2 from participant 2. The N200 component can be observed.

Fig. 5 displayed two comparison plots of the averaged ERP waveforms between target stimuli and non-target stimuli derived from the P300 speller paradigm from participant $2 \& 3$, electrode location $\mathrm{O} 2$ with the G.Tec system. In Fig. 5a, we can distinguish the different amplitudes between the target stimuli waveform and the non-target stimuli waveform in the area from $300 \mathrm{~ms}$ to $400 \mathrm{~ms}$. The peak amplitude in the red trace indicates that the target stimulus has visually registered in the brain. The blue trace has a periodic pattern indicated by the non-target stimulus, this has a similar but smaller response to the target stimulus. It is referred to as the steady-state visual evoked potential (SSVEP) [30]. This diminished signal is in response to the stimulus caused by the change of scene. It has been shown that the non-target stimulus is statistically smaller than the response to the target stimulus. Error bars were plotted at the peak amplitudes of both target and non-target stimuli waveforms. The peak amplitude of target stimuli waveform has standard error $4.46 \pm 0.46 \mathrm{uV}$, the corresponding peak amplitude of the non-target stimuli waveform has a standard error $2.01 \pm 0.20 \mathrm{uV}$. The two error bars did not overlap each other, which denoted that the P300 ERP signal was significant. In Fig. 5b, a large sharp negative peak can be observed in the target stimuli waveform. The waveform contained the N200 component at the peak latency at $220 \mathrm{~ms}$. This signal was well separated from the non-target waveform. To quantitatively evaluate the significance of the N200 component, we calculated the standard error at the negative peak amplitude $7.59 \pm 0.74 \mathrm{uV}$ (target) vs. $-1.16 \pm 0.28 \mathrm{uV}$ (non-target).

\section{(a.) Averaged ERP waveform from subject 3, Gtec, Channel 02}

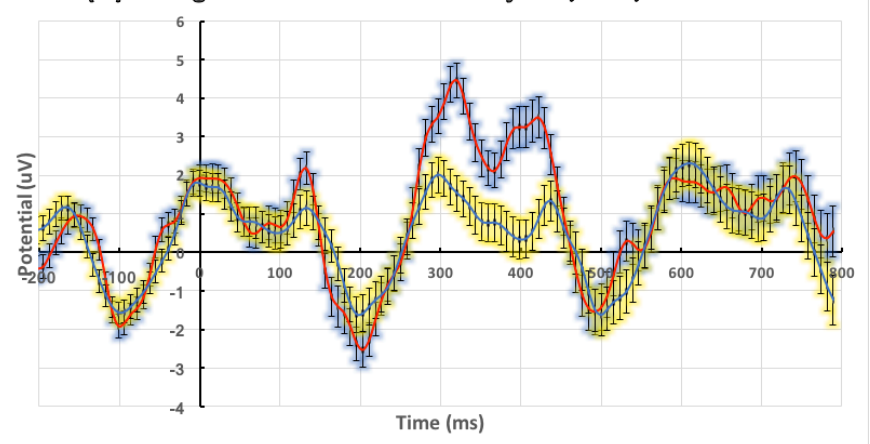

(b.) Averaged ERP waveform from subject 2, Gtec, Channel 02

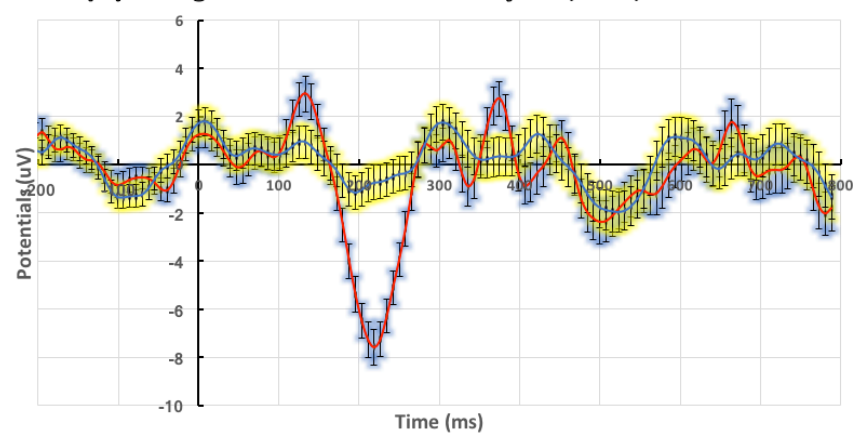

Fig. 5 Averaged ERP waveforms of target and non-target comparison with error bars. RED curve is target stimulus, BLUE curve is non-target stimulus (a.) ERP target waveform and non-target waveform was recorded with G.Tec system from electrodes location $\mathrm{O} 2$ on participant 3. (b.) ERP target waveform and non-target waveform was recorded with G.Tec system from electrodes location $\mathrm{O} 2$ on participant 2. Standard errors were calculated for both target stimuli waveform and non-target stimuli waveform in both (a.) and (b.). 


\section{B. G.Tec and Emotiv features comparison from most active channels}

One of the aims of this paper was to discover the most effective channels to record a P300 ERP signal using the Emotiv system. The electrodes chosen were located at P7 O1 $\mathrm{O} 2$ and P8. These were chosen as the Emotiv EPOC+ headset has only these four electrodes located across the occipital region. The G.Tec system can easily change the electrodes locations associated with the Emotiv system. Table 2 indicates when the error bars do not overlap at the peak point of the P300 and the N200 component. This was done for both the G.Tec system and Emotiv system. The 'tick' represents the error bars at the peak amplitude between target stimuli waveform and non-target stimuli waveform did not overlap. The 'cross' describes the error bar overlapped and indicates a negative attempt to detect an ERP.

Looking down Table 1, the G.Tec system performed perfectly on participant 1 and 3 , but not on participant 2 . This could be in part due to participant 2 having a large N200 elicitation, refer to Fig. 5b; in addition, it is likely to be subject to subject variability. Looking across Table 1, the electrode location $\mathrm{O} 2$, achieved the best result among all electrodes. The result matches what might be expected, that ERPs are likely to be produced in the $\mathrm{O} 1$ and $\mathrm{O} 2$ area due to the Emotiv system lack of electrodes in the central-parietal area. Similar findings can be explored in Table 2. The G.Tec system performed excellently over all participants in detecting N200 component. For the Emotiv system, the $\mathrm{O} 2$ electrode has the best result.

Table 1 Comparison of error bar overlap at the P300 positive peak between target and non-target stimuli for the measurement of G.Tec and Emotiv.

\begin{tabular}{|c|c|c|c|c|c|c|}
\hline & \multicolumn{2}{|c|}{ Participant 1} & \multicolumn{2}{|c|}{ Participant 2} & \multicolumn{2}{|c|}{ Participant 3} \\
\hline Channel & G.Tec & Emotiv & G.Tec & Emotiv & G.Tec & Emotiv \\
\hline P7 & $\checkmark$ & $x$ & $x$ & $x$ & $\checkmark$ & $x$ \\
\hline $\mathrm{O} 1$ & $\checkmark$ & $x$ & $x$ & $\checkmark$ & $\checkmark$ & $x$ \\
\hline $\mathrm{O} 2$ & $\checkmark$ & $x$ & $\checkmark$ & $\checkmark$ & $\checkmark$ & $\checkmark$ \\
\hline P8 & $V$ & $x$ & $x$ & $x$ & $\checkmark$ & $\checkmark$ \\
\hline
\end{tabular}

Table 2 Comparison of error bar overlap at the N200 negative peak between target and non-target stimuli for the measurement of G.Tec and Emotiv.

\begin{tabular}{|c|c|c|c|c|c|c|}
\hline & \multicolumn{2}{|c|}{ Participant 1} & \multicolumn{2}{|c|}{ Participant 2} & \multicolumn{2}{|c|}{ Participant 3} \\
\hline Channel & G.Tec & Emotiv & G.Tec & Emotiv & G.Tec & Emotiv \\
\hline P7 & $\checkmark$ & $x$ & $\checkmark$ & $\checkmark$ & $\checkmark$ & $x$ \\
\hline $\mathrm{O} 1$ & $\checkmark$ & $x$ & $\checkmark$ & $\checkmark$ & $\checkmark$ & $x$ \\
\hline $\mathrm{O} 2$ & $\checkmark$ & $x$ & $\checkmark$ & $\checkmark$ & $\checkmark$ & $\checkmark$ \\
\hline P8 & $\checkmark$ & $x$ & $\checkmark$ & $x$ & $\checkmark$ & $\checkmark$ \\
\hline
\end{tabular}

Note: tick $=$ no overlap; cross $=$ overlapped.

Strong averaged ERP signals identified for the location O2 from the G.Tec system and the Emotiv system are over-plotted for comparison as shown in Fig. 6. The two graphs have similar ERP signal shape between the measurements of the two systems. In terms of the components peak amplitudes, the G.Tec measurement has stronger P300 detection and weaker N200 detection than the Emotiv measurement. In respect of the component peak latency, the P300 peak latencies are alike from the measurement of both systems, the difference within $10 \mathrm{~ms}$. The N200 peak latencies, which the G.tec measurement is leading $39 \mathrm{~ms}$ to the Emotiv measurement.
To quantify the similarity between the two waveforms, we carried out cross correlation statistics studies. The correlation coefficient ranges between -1 to 1 . Values closer to 1 indicate an increasing similarity. We specifically selected a range from $0 \mathrm{~ms}$ to $550 \mathrm{~ms}$ for each compared ERP waveforms to compute the correlation coefficients and the phase shift (lags). This range included the N200 and P300 features, which we were interested. The outcome showed that correlation coefficient $\mathrm{r}=0.5$ and $\mathrm{lag}=125 \mathrm{~ms}$, indicated a good correlation between the two signals. We also calculated the averaged phase shift across the four electrodes for participant 2 (103.51 $\pm 7.48 \mathrm{~ms})$ and participant $3(105.47 \pm 27.43 \mathrm{~ms})$.

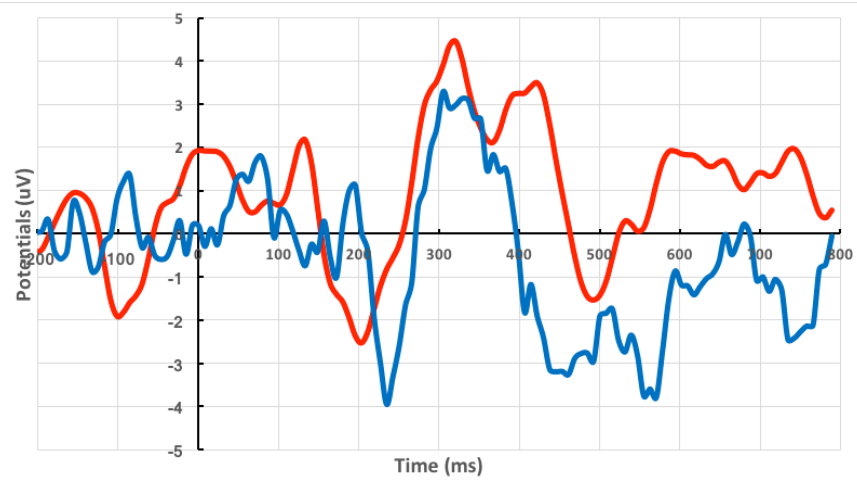

Fig. 6 Comparison of averaged ERPs between the G.Tec and the Emotiv using electrode location O2. The RED curve is the recording from the G.Tec, the BLUE curve is the recording from the Emotiv.

\section{DISCUSSION}

The aim of this paper was to test the validity of the P300 signal measured with the Emotiv EPOC+ system, by comparing the P300 waveforms recorded with a medical grade EEG system, the G.Tec. The results revealed that both N200 and P300 ERP waveforms produced by the EPOC+ system were similar when compared with the waveforms produced by the G.Tec system. Although there are research studies that report that the Emotiv system produces lower quality signals compared to medical grade systems [10, 11, 31-33], this device is still capable of measuring ERP components for non-critical applications, such as visual response or gaming. Our results demonstrate that the Emotiv EPOC + can produce useful ERP waveforms.

The contribution of this study is that the low cost, wireless EEG device has produced measurable ERPs. A limitation of this work has been the small number of participant data, consequently, grand averaged analysis could not be used. However, similar studies of less than five subjects have been published in the past $[10,19,20]$. A more quantitative comparison between the research grade system and the low cost system requires a larger study. A larger study would also be required to understand the variability between subjects. Other factors such as target stimulus sequence, probability, and interstimulus interval may influence the ERPs [34, 35].

The design of the headset with the limited number of electrodes covering the central-parietal area would be another factor limiting the performance of the Emotiv. However, if the electrodes could be relocated by modifying the Emotiv to 
measure the parietal lobe, a better performance may be achieved in future [36].

\section{CONCLUSION}

This study demonstrates that a low-cost wireless EEG system can obtain useful ERP signals when compared with a medical grade EEG system. The N200 and P300 components detection between the medical grade system and the low-cost wireless system are similar. The simplicity of use and increased maneuverability of the wireless system available makes this form of EEG suitable for future work on assessing driver behavior relating to distraction events in a driving simulator with a larger cohort size.

\section{ACKNOWLEDGMENT}

The authors would like to thank Dr. David Malone for providing the RF shielded room at the Hamilton Institute at Maynooth University to do the study. In addition, this work was supported by funding from the Dublin Institute of Technology Fiosraigh Scholarship Programme and the Maynooth Studentship Scheme.

\section{REFERENCES}

[1] D. Zumsteg and H. G. Wieser, "Presurgical evaluation: current role of invasive EEG," Epilepsia, vol. 41, no. s3, 2000.

[2] R. S. Fisher et al., "Epileptic seizures and epilepsy: definitions proposed by the International League Against Epilepsy (ILAE) and the International Bureau for Epilepsy (IBE)," Epilepsia, vol. 46, no. 4, pp. 470-472, 2005

[3] S. Sur and V. K. Sinha, "Event-related potential: An overview," Industrial psychiatry journal, vol. 18, no. 1, p. 70, 2009.

[4] S. J. Luck, An Introduction to the Event-Related Potential Technique (A Bradford book, no. Book, Whole). MIT Press, 2014.

[5] T. C. Handy, Event-related Potentials: A Methods Handbook (A Bradford book, no. Book, Whole). MIT Press, 2005.

[6] J. R. Wolpaw et al., "Brain-computer interface technology: a review of the first international meeting," IEEE transactions on rehabilitation engineering, vol. 8, no. 2, pp. 164-173, 2000.

[7] "Muse - the brain sensing headband." Available: http://www.choosemuse.com/. [Accessed: 05 Feb 2018] "Emotiv - Epoc bain sensing headband." Available: https://www.emotiv.com/. [Accessed: 01 Feb 2018]

"Neurosky - Mindwave brain sensing headband." Available: http://store.neurosky.com/pages/mindwave. [Accessed: 01 Feb $2018]$

[10] A. Campbell et al., "NeuroPhone: brain-mobile phone interface using a wireless EEG headset," 2010, pp. 3-8: ACM.

[11] T. Jijun, Z. Peng, X. Ran, and D. Lei, "The portable P300 dialing system based on tablet and Emotiv Epoc headset," 2015, pp. 566569: IEEE.

[12] S. Debener, F. Minow, R. Emkes, K. Gandras, and M. Vos, "How about taking a low - cost, small, and wireless EEG for a walk?," Psychophysiology, vol. 49, no. 11, pp. 1617-1621, 2012.

[13] D. E. J. Linden, "The P300: where in the brain is it produced and what does it tell us?," The Neuroscientist, vol. 11, no. 6, pp. 563576, 2005.

[14] N. A. Badcock et al., "Validation of the Emotiv EPOC EEG system for research quality auditory event-related potentials in children," PeerJ, vol. 3, p. e907, 2015.

[15] N. A. Badcock, P. Mousikou, Y. Mahajan, P. de Lissa, J. Thie, and G. McArthur, "Validation of the Emotiv EPOC® EEG gaming system for measuring research quality auditory ERPs," PeerJ, vol. 1, p. e38, 2013.

[16] P. de Lissa, S. Sörensen, N. Badcock, J. Thie, and G. McArthur, "Measuring the face-sensitive N170 with a gaming EEG system: A validation study," Journal of Neuroscience Methods, vol. 253, pp. 47-54, 2015/09/30/ 2015

[17] K. Stytsenko, E. Jablonskis, and C. Prahm, "Evaluation of consumer EEG device Emotiv EPOC," in MEi:CogSci Conference, Ljubljana, Slovenia, 2011.

[18] G-TEC. "g.BSamp biosignal amplifier ". Available: http://www.gtec.at/Products/Hardware-and-

Accessories/g.USBamp-Specs-Features. [Accessed: 01 Feb 2018]

[19] C. Ashby, A. Bhatia, F. Tenore, and J. Vogelstein, "Low-cost electroencephalogram (EEG) based authentication," 2011, pp. 442445: IEEE.

[20] B. Choi and S. Jo, "A low-cost EEG system-based hybrid braincomputer interface for humanoid robot navigation and recognition," PloS one, vol. 8, no. 9, p. e74583, 2013.

[21] L. A. Farwell and E. Donchin, "Talking off the top of your head: toward a mental prosthesis utilizing event-related brain potentials," Electroencephalography and Clinical Neurophysiology, vol. 70, no. 6, pp. 510-523, 1988/12/01/1988.

[22] Y. Renard et al., "OpenViBE: an open-source software platform to design, test, and use brain-computer interfaces in real and virtual environments," Presence: teleoperators and virtual environments, vol. 19 , no. 1 , pp. $35-53,2010$.

[23] A. Delorme and S. Makeig, "EEGLAB: an open source toolbox for analysis of single-trial EEG dynamics including independent component analysis," Journal of neuroscience methods, vol. 134, no. 1, pp. 9-21, 2004.

[24] J. Lopez-Calderon and S. J. Luck, "ERPLAB: an open-source toolbox for the analysis of event-related potentials," Frontiers in human neuroscience, vol. 8, 2014

[25] W. Li and C. D. Gilbert, "Perceptual Learning: Neural Mechanisms A2 - Squire, Larry R," in Encyclopedia of NeuroscienceOxford: Academic Press, 2009, pp. 535-541.

[26] S. H. Patel and P. N. Azzam, "Characterization of N200 and P300: selected studies of the event-related potential," International journal of medical sciences, vol. 2, no. 4, p. 147, 2005.

[27] B. Hong, F. Guo, T. Liu, X. Gao, and S. Gao, "N200-speller using motion-onset visual response," Clinical neurophysiology, vol. 120, no. 9, pp. 1658-1666, 2009.

[28] C. Ogura et al., "N200 component of event-related potentials in depression," Biological Psychiatry, vol. 33, no. 10, pp. 720-726, 1993.

[29] S. R. Jackson, G. M. Jackson, and M. Roberts, "The selection and suppression of action: ERP correlates of executive control in humans," Neuroreport, vol. 10, no. 4, pp. 861-865, 1999.

[30] A. M. Norcia, L. G. Appelbaum, J. M. Ales, B. R. Cottereau, and B. Rossion, "The steady-state visual evoked potential in vision research: a review," Journal of vision, vol. 15, no. 6, pp. 4-4, 2015. L. Mayaud et al., "A comparison of recording modalities of P300 event-related potentials (ERP) for brain-computer interface (BCI) paradigm," Neurophysiologie Clinique/Clinical Neurophysiology, vol. 43, no. 4, pp. 217-227, 2013.

[32] C. C. Duncan et al., "Event-related potentials in clinical research: guidelines for eliciting, recording, and quantifying mismatch negativity, P300, and N400," Clinical Neurophysiology, vol. 120, no. 11, pp. 1883-1908, 2009.

[33] M. Duvinage, T. Castermans, M. Petieau, T. Hoellinger, G. Cheron, and T. Dutoit, "Performance of the Emotiv Epoc headset for P300-based applications," Biomedical engineering online, vol. 12 , no. 1 , p. $56,2013$.

[34] J. Polich and T. Bondurant, "P300 Sequence Effects, Probability, and Interstimulus Interval," Physiology \& Behavior, vol. 61, no. 6, pp. 843-849, 1997.

[35] C. J. Gonsalvez and J. Polich, "P300 amplitude is determined by target-to-target interval," Psychophysiology, vol. 39, no. 3, pp. 388-396, 2002.

[36] M. P. Barham, G. M. Clark, M. J. Hayden, P. G. Enticott, R. Conduit, and J. A. G. Lum, "Acquiring research - grade ERPs on a shoestring budget: A comparison of a modified Emotiv and commercial SynAmps EEG system," Psychophysiology, vol. 54, no. 9, pp. 1393-1404, 2017. 